\title{
Antibodies to post-translationally modified insulin as a novel biomarker for prediction of type 1 diabetes in children
}

\author{
Rocky Strollo $^{1}$ • Chiara Vinci ${ }^{1,2} \cdot$ Nicola Napoli $^{1,3}$ Paolo Pozzilli ${ }^{1,4}$. \\ Johnny Ludvigsson ${ }^{5}$ - Ahuva Nissim ${ }^{2}$
}

Received: 7 January 2017 / Accepted: 3 April 2017 /Published online: 20 May 2017

(C) The Author(s) 2017. This article is an open access publication

\begin{abstract}
Aims/hypothesis We have shown that autoimmunity to insulin in type 1 diabetes may result from neoepitopes induced by oxidative post-translational modifications (oxPTM). Antibodies specific to oxPTM-insulin (oxPTM-INS-Ab) are present in most newly diagnosed individuals with type 1 diabetes and are more common than autoantibodies to native insulin. In this study, we investigated whether oxPTM-INS-Ab are present before clinical onset of type 1 diabetes, and evaluated the ability of oxPTMINS-Ab to identify children progressing to type 1 diabetes.

Methods We used serum samples collected longitudinally from the 'All Babies in Southeast Sweden (ABIS)' cohort tested for the gold standard islet autoantibodies to insulin (IAA), GAD (GADA), tyrosine phosphatase 2 (IA-2A) and zinc transporter
\end{abstract}

Paolo Pozzilli, Johnny Ludvigsson and Ahuva Nissim share senior authorship.

Paolo Pozzilli

p.pozzilli@unicampus.it

Ahuva Nissim

a.nissim@qmul.ac.uk

1 Endocrinology \& Diabetes, Università Campus Bio-Medico di Roma, Via Alvaro del Portillo 21, 00128 Rome, Italy

2 Centre for Biochemical Pharmacology, William Harvey Research Institute, Barts and The London School of Medicine and Dentistry, Queen Mary University of London, Charterhouse Square, London EC1M 6BQ, UK

3 I.R.C.C.S. Istituto Ortopedico Galeazzi, Milan, Italy

4 Centre for Immunobiology, the Blizard Institute, Barts and The London School of Medicine and Dentistry, Queen Mary University of London, London, UK

5 Division of Pediatrics, Department of Clinical Experimental Medicine, Medical Faculty, Linköping University, Linköping, Sweden
8 (ZnT8A). We studied 23 children who progressed to type 1 diabetes (progr-T1D) and 63 children who did not progress to type 1 diabetes (NP) after a median follow-up of 10.8 years (interquartile range 7.7-12.8). Of the latter group, 32 were positive for one or more islet autoantibodies $\left(\mathrm{NP}-\mathrm{AAB}^{+}\right)$. oxPTMINS- $\mathrm{Ab}$ to insulin modified by ${ }^{\circ} \mathrm{OH}$ or $\mathrm{HOCl}$ were measured by our developed ELISA platform.

Results Antibodies to at least one oxPTM-INS were present in 91.3\% of progr-T1D children. oxPTM-INS-Ab co-existed with GADA, IA-2A, IAA or ZnT8A in $65.2 \%, 56.5 \%$, $38.9 \%$ and $33.3 \%$ progr-T1D children, respectively. In addition, oxPTM-INS-Ab were present in $17.4 \%, 26.1 \%, 38.9 \%$ and $41.6 \%$ of progr-T1D children who were negative for GADA, IA-2A, IAA and ZnT8A, respectively. ${ }^{\circ} \mathrm{OH}-\mathrm{INS}-\mathrm{Ab}$ were more common in progr-T1D children than in NP-AAB ${ }^{+}$ children $(82.6 \%$ vs $19 \% ; p<0.001)$ and allowed discrimination between progr-T1D and NP-AAB ${ }^{+}$children with $74 \%$ sensitivity and $91 \%$ specificity. None of the NP-AAB ${ }^{-}$children were positive for oxPTM-INS-Ab.

Conclusions/interpretation oxPTM-INS-Ab are present before the clinical onset of type 1 diabetes and can identify children progressing to type 1 diabetes.

Keywords Biomarker · Insulin · Insulin autoantibodies · Islet autoantibodies $\cdot$ Oxidative stress $\cdot$ Post-translational modifications - Type 1 diabetes

$\begin{array}{ll}\text { Abbreviations } & \\ \text { ABIS } & \text { All Babies in Southeast Sweden } \\ \text { GADA } & \text { GAD autoantibodies } \\ \text { GLY-INS } & \text { Glycated insulin } \\ \text { HEL } & \text { Hen egg lysozyme } \\ \text { HOCl-INS } & \text { HOCl-modified insulin } \\ \text { IA-2A } & \text { Tyrosine phosphatase autoantibodies }\end{array}$




\begin{tabular}{|c|c|}
\hline IAA & Insulin autoantibodies \\
\hline${ }^{\circ} \mathrm{OH}-\mathrm{INS}$ & ${ }^{\circ} \mathrm{OH}-$ modified insulin \\
\hline NT-INS & Native insulin \\
\hline охРТM & Oxidative PTM \\
\hline oxPTM-HEL & $\begin{array}{l}\text { Oxidative post-translationally } \\
\text { modified HEL }\end{array}$ \\
\hline oxPTM-INS & $\begin{array}{l}\text { Oxidative post-translationally } \\
\text { modified insulin }\end{array}$ \\
\hline oxPTM-INS-Ab & Antibodies to oxPTM-INS \\
\hline ogr-T1D & Progressing to type 1 diabetes; \\
\hline NP-AAB ${ }^{+}$ & $\begin{array}{l}\text { Autoantibody-positive, non-progressing } \\
\text { to type } 1 \text { diabetes }\end{array}$ \\
\hline NP-AAB ${ }^{-}$ & $\begin{array}{l}\text { Autoantibody-negative, non-progressing } \\
\text { to type } 1 \text { diabetes }\end{array}$ \\
\hline$\Gamma$ & Post-translational modification \\
\hline $3 \mathrm{~A}$ & Radiobinding assay \\
\hline T8A & Zinc transporter 8 autoantibodies \\
\hline
\end{tabular}

\section{Introduction}

Type 1 diabetes is characterised by insulin deficiency and hyperglycaemia due to extensive destruction of insulinproducing beta cells. The autoimmune nature of the disease is suggested by the presence of a pool of circulating autoantibodies against beta cell proteins even years before the clinical onset, such as autoantibodies to insulin (IAA), GAD (GADA), tyrosine phosphatase (IA-2A) and zinc transporter 8 (ZnT8A) [1].

The mechanism underlying the breach of immune tolerance to beta cell antigens is still unclear. Neoepitopes that are post-translationally modified from the native antigens have been recently described in type 1 diabetes [2-7]. Being different from the native proteins, the post-translational modification (PTM) of self-antigens may be recognised as foreign and result in breakdown of tolerance [8-10]. Oxidative stress is a key feature of many autoimmune diseases and results in an excess of reactive oxidants able to generate oxidative PTM (oxPTM) $[4,11]$. Products of oxidative stress are increased in type 1 diabetes [12] and also in individuals at risk [13]. During insulitis, the beta cells that are under stress and the high influx of metabolically active immune cells generate large quantities of reactive oxidants, including the superoxide radical, hydroxyl radical $\left({ }^{\circ} \mathrm{OH}\right)$, hypochlorous acid $(\mathrm{HOCl})$ and peroxynitrate [14]. We have shown that autoimmunity to insulin may result from neoepitopes induced by oxPTM [11]. We found that antibodies specific to oxidative posttranslationally modified insulin (oxPTM-INS) are present in the majority of newly diagnosed individuals with type 1 diabetes and are significantly more abundant than autoantibodies to native insulin (NT-INS) [11].

Islet autoantibodies (IAA, GADA, IA-2A and ZnT8A) represent the most robust approach to identify individuals at risk and to predict progression to clinical disease in those genetically at risk [15] and in the general population [16]. Children with any two autoantibodies may have a risk of $80 \%$ for developing type 1 diabetes during childhood or adolescence [15]. However, multiple antibody testing is required for the best prediction. An additional problem is that radiobinding assays (RBAs), the gold standard for islet autoantibodies, have expensive regulatory requirements. These elements introduce additional complexity that may limit the implementation of autoantibody screening. Finally, a significant number of individuals test negative to these markers [17]. Therefore, the development of alternative technologies remains an unmet need. To address this we have developed an ELISA that detects auto-reactivity to oxPTM-INS. We showed that the assay detecting antibodies to oxPTM-INS (oxPTM-INS-Ab) is highly accurate (84\% sensitivity, 99\% specificity), may detect over $30 \%$ of individuals who are negative to the RBA for IAA and, when combined with IAA, identifies $95 \%$ of individuals with newly diagnosed type 1 diabetes [11]. However, the predictive potential of oxPTMINS-Ab is not known yet.

We hypothesise that oxPTM-INS-Ab are present before the clinical onset of the disease. To test this hypothesis, considering the high prevalence of oxPTM-INS reactivity in newly diagnosed type 1 diabetes, we evaluated the ability of this novel autoantibody specificity to identify children progressing to clinical disease. We used serum samples from the ABIS (All Babies in Southeast Sweden) study, a large prospective study in which unselected children from the general population born during 1997-1999 have been followed prospectively with regular evaluation of islet autoantibodies for the development of type 1 diabetes $[18,19]$.

\section{Methods}

Participants and serum samples Serum samples from the ABIS study were obtained and analysed in a blinded fashion for detection of oxPTM-INS-Ab. The ABIS study is a prospective population-based follow-up study which included 17,055 unselected children born between 1 October 1997 and 1 October 1999 in southeast Sweden [18]. Of the screened children, 116 developed type 1 diabetes during the follow-up. In the present study, we tested 51 samples from the 23 children progressing to type 1 diabetes (progr-T1D), collected longitudinally before diagnosis at three different time points (at the ages of 5, 7 and 11 years). Samples were selected where sufficient serum and autoantibody data were available for this study. Only one time point was available from seven progr-T1D children before the collection at the second time point. As controls, we used samples from 63 children of similar age and sex who did not progress to type 1 diabetes over time, including 64 samples from 32 autoantibody-positive children (autoantibody-positive, nonprogressing to type 1 diabetes $\left[\mathrm{NP}-\mathrm{AAB}^{+}\right]$) and 31 samples from 
autoantibody-negative children (autoantibody-negative, nonprogressing to type 1 diabetes $\left[\mathrm{NP}-\mathrm{AAB}^{-}\right]$). NP-AAB ${ }^{+}$children were defined as positive to at least one islet-antibody marker (IAA, GADA, IA-2A or ZnT8A). Informed consent was obtained from parents prior to the collection of blood. The study was approved by the Research Ethics Committees of the Medical Faculties of Linköping University, Linköping and Lund University, Lund, Sweden and by the Ethics Committee of the Università Campus Bio-Medico di Roma.

ELISA for detection of oxPTM-INS-Ab Insulin was chemically modified as previously described to generate oxPTM-INS modified by $\mathrm{HOCl},{ }^{\circ} \mathrm{OH}$ and glycation using ribose [11]. Hen egg lysozyme (HEL; Sigma-Aldrich, Milan, Italy) was similarly modified and used as a control antigen. oxPTM-INS encompasses glycated (GLY-INS), ${ }^{\circ} \mathrm{OH}-$ modified ('OH-INS) and $\mathrm{HOCl}$-modified insulin (HOCl-INS). An ELISA was performed using NT-INS, oxPTM-INS, control native HEL or control oxidative post-translationally modified HEL (oxPTM-HEL) as targets. Development and calibration of the ELISA is described in our previous publication [11]. ELISA plates (Nunc, London, UK) were coated with $10 \mu \mathrm{g} / \mathrm{ml}$ of modified or native protein in $0.05 \mathrm{~mol} / \mathrm{l}$ carbonate/bicarbonate buffer $(\mathrm{pH} 9.6)$ at $4^{\circ} \mathrm{C}$ overnight. Plates were then washed three times with PBS. After blocking for $2 \mathrm{~h}$ with 5\% BSA in $0.5 \%$ Tween PBS, $100 \mu \mathrm{l}$ of 1:200-diluted serum samples in 5\% BSA in $0.5 \%$ Tween PBS were added to each well, followed by $2 \mathrm{~h}$ incubation at room temperature. Plates were then washed with PBS plus $0.1 \%$ Tween, followed by three washes with PBS. Antihuman IgG-horseradish peroxidase-conjugated antibodies (Sigma-Aldrich) were then added at 1:1000 dilution in 5\% BSA in $0.5 \%$ Tween PBS for another $2 \mathrm{~h}$ incubation. The ELISA plates were washed, and $100 \mu \mathrm{g} / \mathrm{ml} \mathrm{3,3',5,5^{ \prime } -}$ tetramethylbenzidine substrate (Sigma-Aldrich) in $100 \mathrm{mmol} / \mathrm{l}$ sodium acetate ( $\mathrm{pH}$ 6.0), was added. Subsequently, the reaction was stopped with $1 \mathrm{~mol} / \mathrm{l}$ sulphuric acid. The absorbance was measured at $450 \mathrm{~nm}$ using a GENios plate reader and Magellan software (Tecan, Reading, UK). The ELISA absorbance values obtained for HEL and oxPTM-HEL were used as background controls that were subtracted from the absorbance values of NTINS and oxPTM-INS, respectively. In addition, to account for assay fluctuation, binding to insulin, oxPTM-INS, HEL and oxPTM-HEL was tested for each individual sample on the same plate. Each assay included known positive or negative reference control samples. Longitudinal samples obtained from the same individuals were tested on the same plate. Levels of oxPTMINS-Ab above the 99th percentile of 88 healthy individuals were defined as ELISA cut-off. Intra-assay $\mathrm{CV}$ was $<8 \%$ $(n=10)$. Inter-assay CVs were $<10 \%$ and $<13 \%$ for NT-INS and oxPTM-INS-Ab $(n=12)$, respectively.

Islet autoantibodies Islet autoantibodies were measured by RBA. IAA were measured according to the method of Williams et al [20], with some modifications [21]. GADA and IA-2A were measured as previously described by Wahlberg et al [19] and ZnT8A (variants ZnT8RA, ZnT8QA and ZnT8WA) were measured as described by Vaziri-Sani et al [22]. Thresholds have been defined as equivalent to the 98th percentile for GADA, IA-2A and ZnT8A, and the 95th percentile for IAA [23]. In the 2005 Diabetes Autoantibody Standardization Programme (DASP), the RBAs for GADA, IA-2A and IAA achieved $76 \%, 72 \%$ and $28 \%$ sensitivity, respectively, with $96 \%, 100 \%$ and $100 \%$ specificity. In the 2013 Islet Autoantibody Standardization Programme (IASP), the RBA for ZnT8AR, ZnT8AQ and ZnT8WA achieved 50\%, 18\% and 36\% sensitivity, respectively, with 99\%, 100\% and 93\% specificity.

HLA genotyping HLA typing and subtyping was performed as previously described [24]. The HLA genotyping revealed the haplotypes HLA-DQB1, $-D Q A 1$ and $-D R B 1$ and these were categorised as susceptibility-associated $(\mathrm{S})$, neutral $(\mathrm{N})$ and protective $(\mathrm{P})$, according to Hermann et al [24]. Susceptibilityassociated haplotypes included DR4-DQ8 (DRB1*0401/2/4/5$D Q B 1 * 0302)$ and $D R 3-D Q 2(D Q A 1 * 05-D Q B 1 * 02)$, protective haplotypes included $D R 2-D Q 6(D Q B 1 * 0602), D R 11 / 12$ / $1303-D Q 7(D Q A 1 * 05-D Q B 1 * 0301), D R 7-D Q 3$ (DQA1*0201-DQB1*0303), DR14-DQ5 (DQB1*0503), DR403-DQ8 (DRB1*0403-DQB1*0302) and DR1301-DQ6 $(D Q B 1 * 0603)$. Other haplotypes were defined as neutral.

Statistical analysis Statistical analyses were performed using Prism Software version 6.01 (GraphPad, San Diego, CA, USA). Differences in antibody levels between groups were tested by the Mann-Whitney test. Longitudinal changes in antibody binding were evaluated by the Wilcoxon paired test. To determine predictive discrimination between progr-T1D and control groups, we used the 99th percentile of the healthy individuals as cut-off point absorbance units to construct a contingency table of positive oxPTM-INS-Ab against clinical diagnosis and tested it by Fisher's Exact Test.

\section{Results}

Features of the studied population The characteristics of the study population are shown in Table 1 . At the earliest time point studied, the progr-T1D, NP-AAB ${ }^{+}$and NP-AAB groups were comparable in terms of sex, while age was slightly higher in the NP-AAB ${ }^{+}$group $(p=0.04)$. The majority of progr-T1D children $(n=18,78 \%)$ had multiple positive islet autoantibodies; two were single-positive. Two children were negative for IAA, GADA and IA-2A; one child was negative for GADA and IA-2A (IAA was not assessed). Only $25 \%$ $(n=8)$ of NP-AAB ${ }^{+}$children had multiple positive islet autoantibodies. HLA typing was available in 43 children; HLA susceptible phenotypes were more common in progr-T1D 
Table 1 Characteristics of the study population

\begin{tabular}{llll}
\hline Characteristics & Progr-T1D $(N=23)$ & \multicolumn{2}{l}{ Children not progressing to type 1 diabetes } \\
\cline { 3 - 4 } & & $\operatorname{NP}^{-A_{A B}}(N=32)$ & NP-AAB $^{-}(N=31)$ \\
\hline Age at baseline, years & $6.17 \pm 1.49$ & $7.61 \pm 2.42$ & $7.13 \pm 2.07$ \\
Sex, male & $15(65)$ & $21(66)$ & $17(55)$ \\
Multiple autoantibodies $(\geq 2)$ & $18(78)$ & $8(25)$ & NA \\
\hline
\end{tabular}

Data are presented as means $\pm \mathrm{SD}$ or $n(\%)$

The three groups were comparable in terms of sex, although age was slightly higher in the NP-AAB ${ }^{+}$group than in the progr-T1D group $(p=0.04)$. Eighteen ( $78 \%)$ of progr-T1D and $8(25 \%)$ of NP-AAB ${ }^{+}$children had multiple positive islet autoantibodies than in NP-AAB ${ }^{+}$and NP-AAB ${ }^{-}$children $(p<0.007)$, while the prevalence of protective phenotypes was similar between the three groups $(p>0.07)$ (Table 2). In the progr-T1D group, median follow-up from the first sample analysed to diagnosis was 5.1 years (interquartile range 3.2-7.7). After a median follow-up of 10.8 years (interquartile range $7.7-12.8$ ), all children in the control groups, either NP-AAB ${ }^{+}$or NP-AAB ${ }^{-}$, remained free from diabetes (last assessment performed in December 2016).

\section{Cross-sectional evaluation of oxPTM-INS-Ab prevalence} Binding and prevalence of oxPTM-INS-Ab were compared between progr-T1D, NP-AAB ${ }^{+}$and NP-AAB ${ }^{-}$children at the earliest time point available. In the progr-T1D group, binding to $\mathrm{HOCl}-\mathrm{INS}$ and ${ }^{\circ} \mathrm{OH}-\mathrm{INS}$ was significantly higher than binding to NT-INS ( $p<0.001$; Fig. 1a). Serum samples from $21(91.3 \%)$ progr-T1D children bound to at least one oxPTMINS ('OH-INS or HOCl-INS) and 17 (73.9\%) children were

Table 2 HLA-genotypes prevalence in the study population

\begin{tabular}{llll}
\hline $\begin{array}{l}\text { HLA susceptibility } \\
\text { category }\end{array}$ & $\begin{array}{l}\text { Progr-T1D } \\
(N=17)\end{array}$ & $\begin{array}{l}\text { Children not progressing to } \\
\text { type 1 diabetes }\end{array}$ \\
\cline { 3 - 4 } & & $\begin{array}{l}\text { NP-AAB } \\
(N=16)\end{array}$ & $\begin{array}{l}\text { NP-AAB }^{-} \\
(N=10)\end{array}$ \\
\hline SS & $8(47)$ & $3(18.75)$ & $0(0)$ \\
SN & $6(35)$ & $3(18.75)$ & $1(10)$ \\
SP & $3(18)$ & $4(25)$ & $4(40)$ \\
NN & $0(0)$ & $2(12.5)$ & $2(20)$ \\
NP & $0(0)$ & $2(12.5)$ & $2(20)$ \\
PP & $0(0)$ & $2(12.5)$ & $1(10)$ \\
\hline
\end{tabular}

Data are presented as $n(\%)$

HLA haplotypes were available in 43 children and were categorized into susceptibility-associated $(\mathrm{S})$, neutral $(\mathrm{N})$ and protective $(\mathrm{P})$ groups according to Hermann et al. [24]. Susceptibility-associated haplotypes included $D R 4-D Q 8(D R B 1 * 0401 / 2 / 4 / 5-D Q B 1 * 0302)$ and $D R 3-D Q 2$ $(D Q A 1 * 05-D Q B 1 * 02)$, protective haplotypes included $D R 2-D Q 6$ (DQB1*0602), DR11/12/1303-DQ7 (DQA1*05-DQB1*0301), DR7DQ3 (DQA1*0201-DQB1*0303), DR14-DQ5 (DQB1*0503), DR403$D Q 8(D R B 1 * 0403-D Q B 1 * 0302)$ and DR1301-DQ6 $(D Q B 1 * 0603)$. Other haplotypes were defined as neutral found to be double-positive to both ${ }^{\circ} \mathrm{OH}-\mathrm{INS}$ and HOCl-INS. Binding to HOCl-INS and ${ }^{\circ} \mathrm{OH}-\mathrm{INS}$ was higher in sera from progr-T1D children compared with either NP-AAB ${ }^{+}$or $\mathrm{NP}^{-\mathrm{AAB}^{-}}$children $(p<0.001$; Fig. 1a). oxPTM-INS-Ab were more common in progr-T1D children than in NP-AAB ${ }^{+}$children, with respective reactivity to ${ }^{\circ} \mathrm{OH}-\mathrm{INS}$ in $82.6 \%(19 / 23)$ vs $19 \%(6 / 32)(p<0.0001)$ and reactivity to HOCl-INS in $82.6 \%(19 / 23)$ vs $40.6 \%(13 / 32)(p=0.0024)$ (Fig. $1 b-d)$. OH-INS-Ab allowed discrimination between progr-T1D and NP-AAB ${ }^{+}$children with $74 \%$ sensitivity and $91 \%$ specificity. The overlap between NT-INS and oxPTM-INS-Ab is shown in Fig. 1b-d. Binding to NT-INS was more common in progr-T1D children than in NT-AAB ${ }^{+}$and NT-AAB ${ }^{-}$children $(p<0.001)$. Binding to both NT-INS and oxPTM-INS occurred in $14(60 \%)$ progr-T1D children, while 7 (30\%) displayed binding only to oxPTM-INS. In the NP-AAB ${ }^{+}$children, $6(19 \%)$ displayed binding to both NT-INS and oxPTM-INS, while 7 (22\%) displayed binding only to oxPTM-INS. None of the NP-AAB ${ }^{-}$children were positive for oxPTM-INS-Ab.

Longitudinal changes of oxPTM-INS-Ab in prog-T1D children Binding to oxPTM-INS (either ${ }^{\circ} \mathrm{OH}-\mathrm{INS}$ or $\mathrm{HOCl}-\mathrm{INS}$ ) did not change significantly over time (median follow-up 3 years [range 3-6 years]; $p=0.725$; Fig. 2a). Seroconversion occurred in four children: two became positive at a later stage while two became negative. When evaluated according to time before diabetes onset, reactivity to oxPTMINS appeared as early as 11 years before disease onset (median time to diabetes onset 6 years [range $2-11$ years]; Fig. $2 b$ ).

Distribution of oxPTM-INS-Ab and islet autoantibodies Figure 3 shows the degree of overlap between oxPTM-INS-Ab (OH-INS-Ab) and other islet autoantibodies in progr-T1D and NP-AAB ${ }^{+}$children. IAA data were available in 18 progr-T1D and $27 \mathrm{NP}-\mathrm{AAB}^{+}$children and $\mathrm{ZnT} 8 \mathrm{~A}$ data were available in 12 progr-T1D and $10 \mathrm{NP}-\mathrm{AAB}^{+}$children. While binding and prevalence of oxPTM-INS-Ab were significantly higher in progrT1D children than in NP-AAB ${ }^{+}$children $(p<0.001)$, both titres and prevalence of GADA, IAA and ZnT8A were similar between the two groups $(p>0.257)$. IA-2A was the most specific 

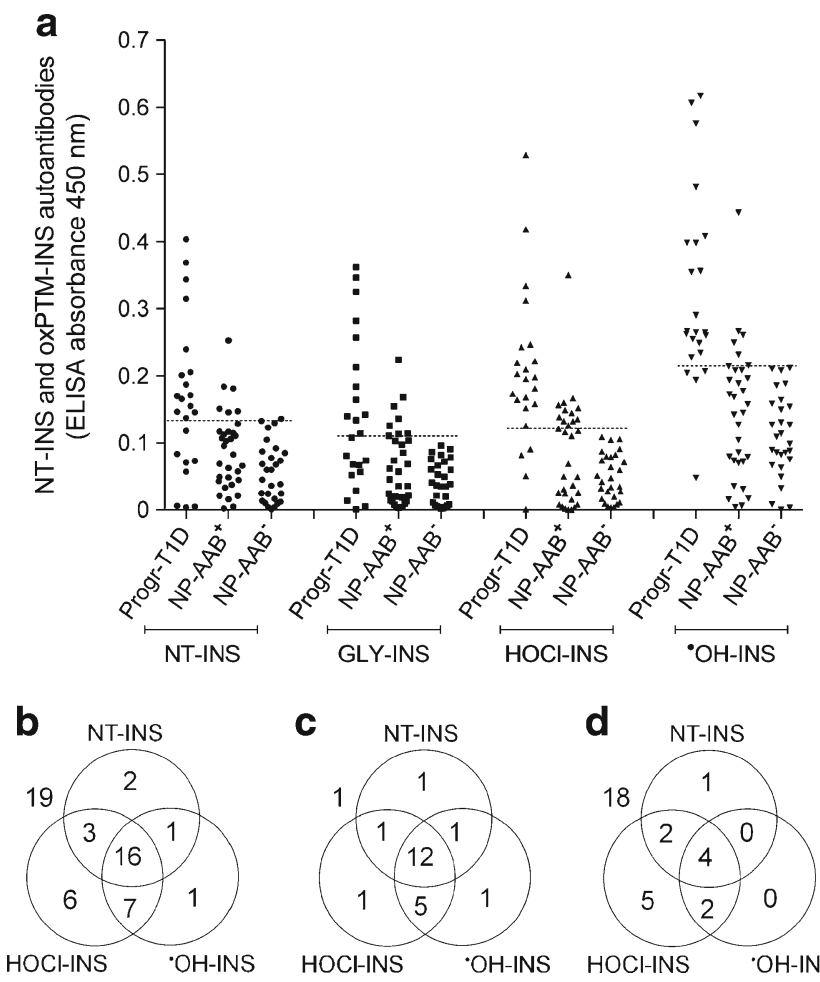

Fig. 1 Cross-sectional evaluation of antibody binding to oxPTM-INS in study population. (a) Reactivity to NT-INS and oxPTM-INS was significantly higher in samples from progr-T1D children compared with nonprogressing children, regardless of whether they were NP-AAB ${ }^{+}$or NP$\mathrm{AAB}^{-}$to the standard islet autoantibody markers $(p<0.001)$. Binding to oxPTM-INS modified by $\mathrm{HOCl}$ and ${ }^{\circ} \mathrm{OH}$ was significantly higher than to NT-INS in progr-T1D children $(p<0.0001)$. Data on the earliest time point available are reported. Values above the dashed lines were defined as positive for antibodies to NT-INS and oxPTM-INS modified by glycation (GLY), $\mathrm{HOCl}$ or ${ }^{\circ} \mathrm{OH}$, respectively (99th percentile of a group of 88 healthy control children). (b-d) Overlapping prevalence of antibodies to NT-INS and oxPTM-INS in all children positive for at least one islet autoantibody (b) and in progr-T1D (c) and NP-AAB ${ }^{+}$children (d). Values outside the circles are children negative for the antibody evaluated in the diagram

marker among autoantibodies evaluated, being significantly higher in the progr-T1D group than in the NP-AAB ${ }^{+}$group $(p<0.001)$. In the progr-T1D group, $19(82.6 \%)$ children were positive to ${ }^{\circ}$ OH-INS-Ab, 18 (78.3\%) to GADA, $16(69.5 \%)$ to IA-2A, $10(55.5 \%)$ to IAA and 6 (50\%) to ZnT8A (Fig. 3e-h). oxPTM-INS-Ab co-existed with GADA, IA-2A, IAA or ZnT8A in $15 / 23(65.2 \%), 13 / 23(56.5 \%), 7 / 18(38.9 \%)$ and $4 / 12(33.3 \%)$
progr-T1D children (Fig. 3e-h). In addition, oxPTM-INS-Ab detected 4/23 (17.4\%), 6/23 (26.1\%), 7/18 (38.9\%) and 5/12 (41.6\%) progr-T1D children who were negative for GADA, IA-2A, IAA and ZnT8A, respectively (Fig. 3e-h). Two progrT1D children were negative for GADA, IA-2A and IAA (ZnT8A not assessed) but showed positive for oxPTM-INS$\mathrm{Ab}$. The assessment of oxPTM-INS-Ab in combination with IA-2A and IAA led to the identification of $100 \%$ progr-T1D children with the lowest percentage of false-positive results among the other possible antibody combinations (Fig. 4). In the NP-AAB ${ }^{+}$group, oxPTM-INS-Ab reactivity was similar in those who were positive to one or more standard islet autoantibodies $(p>0.148)$.

\section{Discussion}

We have recently shown that oxPTM-INS-Ab are very common in newly diagnosed type 1 diabetes, being detected in $84 \%$ of individuals [11]. In this study, we found that oxPTM-INS auto-reactivity is present before diabetes diagnosis in over $90 \%$ of individuals. oxPTM-INS-Ab could discriminate between prog-T1D children and those who did not progress to type 1 diabetes regardless of positivity to other islet autoantibodies. Therefore, these data may suggest a potential role for oxPTM-INS-Ab as a predictive biomarker of type 1 diabetes.

The main strength of our study is the prospective evaluation of oxPTM-INS-Ab in a well-characterised cohort of children from the general population, tested for the standard diabetes autoantibodies. The uniqueness of the ABIS study resides in the possibility of applying its findings to the general population, since participants were not selected according to predetermined diabetes risk (genetic or familial). An additional strength was that the long follow-up in some progr-T1D children allowed us to discover whether oxPTM-INS-Ab develop very early in the natural history of the disease. Our study has limitations. The earliest time point tested in the progr-T1D children was at 5 years of age. Data from birth cohorts suggest that peak age of seroconversion in high-risk individuals is around 2 years of age [25]. Therefore, we cannot exclude
Fig. 2 Longitudinal changes of oxPTM-INS-Ab in prog-T1D children according to age (a) and time before diagnosis of type 1 diabetes (b). Values above the dashed lines were defined as positive for antibodies to oxPTMINS; ${ }^{\circ} \mathrm{OH}-\mathrm{INS}$ is shown as an example for oxPTM-INS-Ab
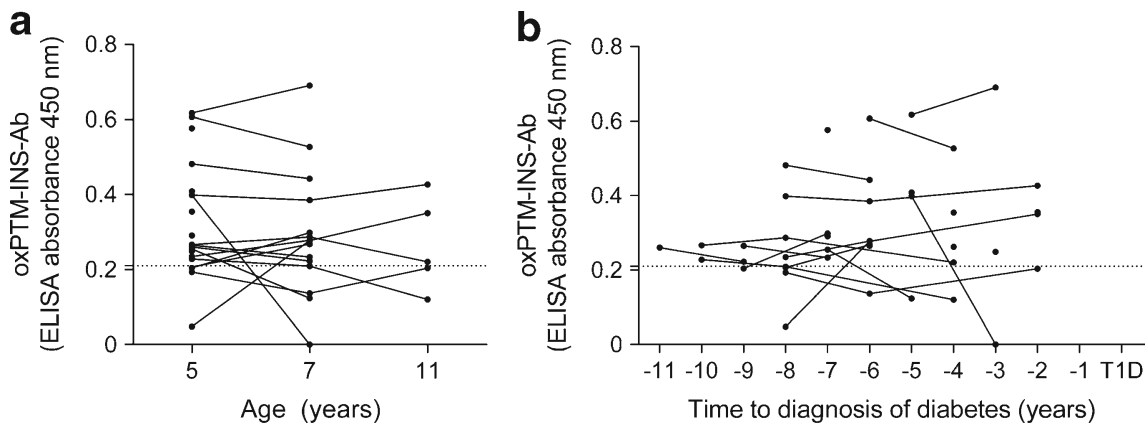
Fig. 3 Overlapping prevalence of oxPTM-INS-Ab, GADA, IA2A, IAA and ZnT8A. Data are shown for the whole study population of children positive to d) and for progr-T1D (e-h) and NP-AAB ${ }^{+}$children (i-l). Data for IAA and ZnT8A were available in 45 and 22 children, respectively. ZnT8A included positivity to one or more ZnT8RA, ZnT8AWA and ZnT8QA variants. $\mathrm{OH}-\mathrm{INS}$ is shown as example for oxPTMINS-Ab. Values outside the antibody evaluated in the diagram at least one islet autoantibody (acircles are children negative to the a

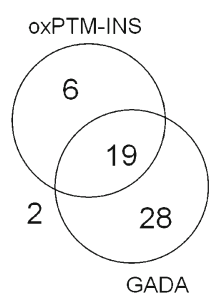

e

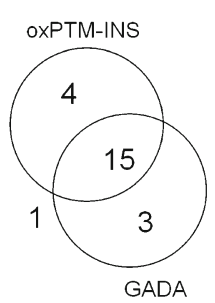

i

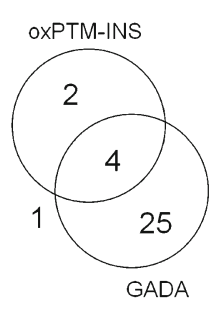

b

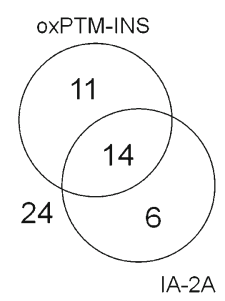

f

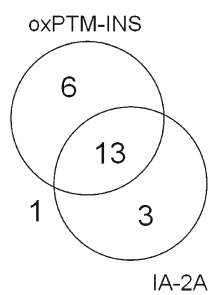

\section{j}

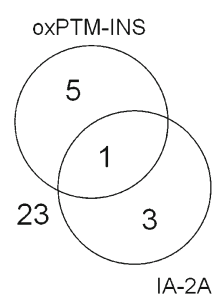

C

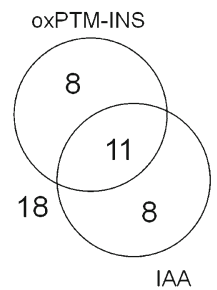

g

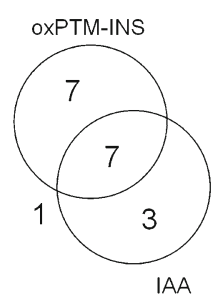

k

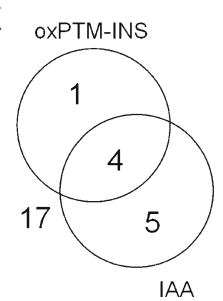

d oxPTM-INS

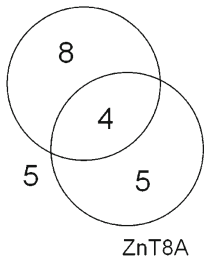

h

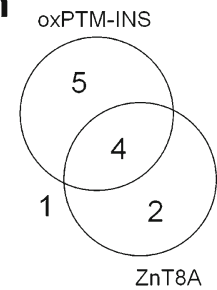

I OXPTM-INS

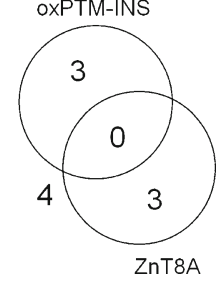

the possibility that children defined as negative for the standard islet autoantibodies have not previously been seropositive for one or more islet autoantibodies. A second limitation is that the determination of ZnT8A was performed on a limited number of children, as ZnT8A assays became available only after the follow-up for many children in the ABIS study.

Our findings shed further light on type 1 diabetes pathogenesis. Detection of oxPTM-INS reactivity before clinical onset of of oxPTM-INS-Ab, GADA, IA$2 \mathrm{~A}$ and IAA evaluated with three standard islet autoantibodies or with oxPTM-INS-Ab substituted for GADA, IA-2A or IAA. Data are shown for the whole study population of children positive to at least one islet autoantibody (ad) and for progr-T1D (e-h) and $\mathrm{NP}-\mathrm{AAB}^{+}$children $(\mathbf{i}-\mathbf{l})$. IAA was not available in five progr-T1D and five NP-AAB ${ }^{+}$children; therefore these children are not included in the diagrams assessing the overlapping prevalence of IAA. ${ }^{\circ} \mathrm{OH}-\mathrm{INS}$ is shown as example for oxPTMINS-Ab. Values outside the circles are children negative to the antibodies evaluated in the diagram
Fig. 4 Overlapping prevalence a

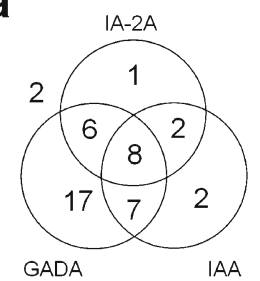

e

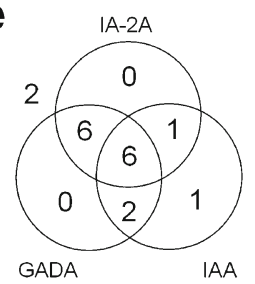

i

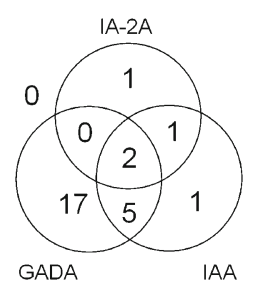

b

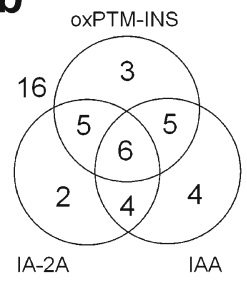

f
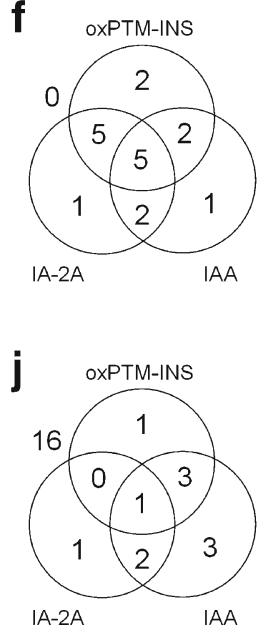

C
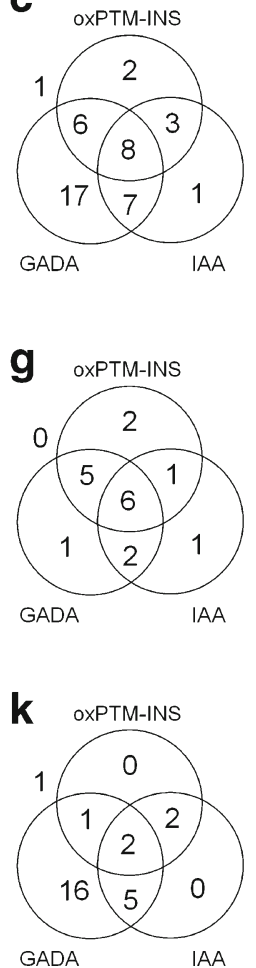

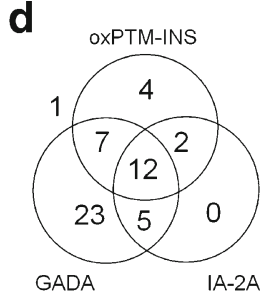

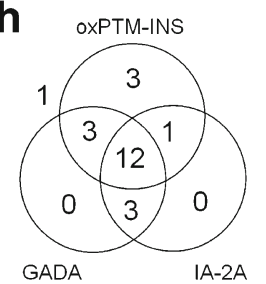

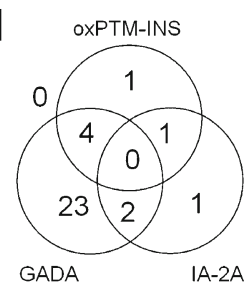


type 1 diabetes is consistent with evidence that the unbalanced redox state takes place early in the natural history of the disease. Individuals with a short disease duration have an early impairment in antioxidant capacity and over threefold increased levels of lipid peroxidation regardless of blood glucose control [12]. An impaired oxidation status anticipates dysglycaemia; it has been shown that plasma malondialdehyde and erythrocyte malondialdehyde, two markers of oxidative stress, are abnor$\mathrm{mal}$ in euglycaemic individuals at increased risk for type 1 diabetes compared with individuals not at risk [13]. These data, together with our findings, may support the involvement of oxidative stress in type 1 diabetes pathogenesis.

To our knowledge, this is the first study investigating antibody reactivity to oxPTM/PTM of a beta cell antigen as a predictive biomarker of type 1 diabetes. Therefore, a direct comparison with prediction potential of other modified antigens is not possible. Our data are consistent with previous finding by our group in newly diagnosed individuals with type 1 diabetes. Similar to our previous study [11], oxPTM-INS-Ab identified over $80 \%$ of individuals with the disease and were able to detect more than one-third of those who tested negative to the IAA assay. Additional neoepitopes derived from PTM of beta cell autoantigens have been described in humans and in animal models of type 1 diabetes, including antibody response to oxidised GAD [26] and T cell reactivity to GAD citrullination [7], C-peptide deamination [5] and hybrid fused peptides [2, 27]. The response to modified antigens may also involve proteins that are not proper to beta cells $[4,28]$, especially in the presence of defined genetic background. An example is the identification of antibodies to oxPTM-collagen type II in a large proportion of individuals carrying $H L A-D R B 1 * 04$ [4]. In this regard, the extracellular matrix surrounding beta cells [29], or other tissues attacked by autoimmune response in type 1 diabetes (thyroid, gut, joints, etc) [30], may become additional potential targets of oxPTM $[4,31]$. Often such PTM forms induce a more pronounced immune reactivity than the native antigen.

Our results may also have implications for disease prediction and staging. Consistent with previous findings [15, 32], we found low predictive accuracy of GADA and IAA when analysed as a single test, while IA-2A showed a highly specific association with type 1 diabetes progression [15]. A main finding of our study is the predictive accuracy of oxPTM-INS-Ab. Of note, oxPTM-INS-Ab identified individuals with preclinical disease otherwise classified as antibody-negative or single-positive. As highlighted by a recent statement by the JDRF, Endocrine Society and ADA, islet autoimmunity (as defined by the presence of two or more islet autoantibodies) represents the earliest stage of type 1 diabetes and identifies a target population for prevention trials and future preventive strategies [33]. If confirmed in larger studies, oxPTM-INS-Ab may be adopted as an additional biomarker to further redefine disease taxonomy, allowing better prediction and therefore better stratification into eligibility trials.
In conclusion, we showed that immune reactivity to oxPTM-INS is present before clinical onset of type 1 diabetes and that measurement of oxPTM-INS-Ab may identify children likely to progress to type 1 diabetes. This is the first evidence suggesting that oxPTM of a beta cell autoantigen precedes diabetes onset in humans and that auto-reactivity to oxPTM may act as a predictive biomarker of the disease. Additional studies with larger cohorts are required to confirm the predictive potential of oxPTM-INS-Ab in type 1 diabetes.

Data availability The data are available on request from the authors.

Funding This study was supported by the EFSD/JDRF/Lilly European Programme in Type 1 Diabetes Research (3-PAR-2016-277-A-N) and by the JDRF innovative grant (INO-2015-78-S-B). ABIS and the autoantibody determinations were supported by the Swedish Research Council (K2005-72X-11242-11A and K2008-69X-20826-01-4), the Swedish Child Diabetes Foundation (Barndiabetesfonden), JDRF Wallenberg Foundation (K 98-99D-12813-01A), Medical Research Council of Southeast Sweden (FORSS) and the Swedish Council for Working Life and Social Research (FAS2004-1775).

Duality of interest The authors declare that there is no duality of interest associated with this manuscript.

Contribution statement RS, PP, JL and AN conceived the study. CV contributed to acquisition and analysis of data. NN contributed to data acquisition. RS analysed the data and wrote the first draft. All authors critically revised the manuscript for intellectual content. All authors have seen and approved the final draft. RS is the guarantor of this work and, as such, had full access to all the data in the study and takes responsibility for the integrity of the data and the accuracy of the data analysis.

Open Access This article is distributed under the terms of the Creative Commons Attribution 4.0 International License (http:// creativecommons.org/licenses/by/4.0/), which permits unrestricted use, distribution, and reproduction in any medium, provided you give appropriate credit to the original author(s) and the source, provide a link to the Creative Commons license, and indicate if changes were made.

\section{References}

1. Knip M, Siljander H, Ilonen J, Simell O, Veijola R (2016) Role of humoral beta-cell autoimmunity in type 1 diabetes. Pediatr Diabetes 17(Suppl 22):17-24

2. Delong T, Wiles TA, Baker RL et al (2016) Pathogenic CD4 T cells in type 1 diabetes recognize epitopes formed by peptide fusion. Science 351:711-714

3. Babon JA, DeNicola ME, Blodgett DM et al (2016) Analysis of self-antigen specificity of islet-infiltrating $\mathrm{T}$ cells from human donors with type 1 diabetes. Nat Med 22:1482-1487

4. Strollo R, Rizzo P, Spoletini M et al (2013) HLA-dependent autoantibodies against post-translationally modified collagen type II in type 1 diabetes mellitus. Diabetologia 56:563-572

5. van Lummel M, Duinkerken G, van Veelen PA et al (2014) Posttranslational modification of HLA-DQ binding islet autoantigens in type 1 diabetes. Diabetes 63:237-247 
6. Rondas D, Crevecoeur I, D Hertog W et al (2015) Citrullinated glucose-regulated protein 78 is an autoantigen in type 1 diabetes. Diabetes 64:573-586

7. McGinty JW, Chow IT, Greenbaum C, Odegard J, Kwok WW, James EA (2014) Recognition of posttranslationally modified GAD65 epitopes in subjects with type 1 diabetes. Diabetes 63: 3033-3040

8. Ryan BJ, Nissim A, Winyard PG (2014) Oxidative posttranslational modifications and their involvement in the pathogenesis of autoimmune diseases. Redox Biol 2:715-724

9. Strollo R, Ponchel F, Malmstrom V et al (2013) Autoantibodies to posttranslationally modified type II collagen as potential biomarkers for rheumatoid arthritis. Arthritis Rheum 65:1702-1712

10. Doyle HA, Mamula MJ (2012) Autoantigenesis: the evolution of protein modifications in autoimmune disease. Curr Opin Immunol 24:112-118

11. Strollo R, Vinci C, Arshad MH et al (2015) Antibodies to posttranslationally modified insulin in type 1 diabetes. Diabetologia 58:2851-2860

12. Marra G, Cotroneo P, Pitocco D et al (2002) Early increase of oxidative stress and reduced antioxidant defenses in patients with uncomplicated type 1 diabetes: a case for gender difference. Diabetes Care 25:370-375

13. Matteucci E, Giampietro O (2000) Oxidative stress in families of type 1 diabetic patients. Diabetes Care 23:1182-1186

14. Newsholme P, Rebelato E, Abdulkader F, Krause M, Carpinelli A, Curi R (2012) Reactive oxygen and nitrogen species generation, antioxidant defenses, and $\beta$-cell function: a critical role for amino acids. J Endocrinol 214:11-20

15. Ziegler AG, Rewers M, Simell O et al (2013) Seroconversion to multiple islet autoantibodies and risk of progression to diabetes in children. JAMA 309:2473-2479

16. Knip M, Korhonen S, Kulmala P et al (2010) Prediction of type 1 diabetes in the general population. Diabetes Care 33:1206-1212

17. Wenzlau JM, Juhl K, Yu L et al (2007) The cation efflux transporter $\mathrm{ZnT} 8$ (Slc30A8) is a major autoantigen in human type 1 diabetes. Proc Natl Acad Sci U S A 104:17040-17045

18. Ludvigsson J, Ludvigsson M, Sepa A (2001) Screening for prediabetes in the general child population: maternal attitude to participation. Pediatr Diabetes 2:170-174

19. Wahlberg J, Vaarala O, Ludvigsson J (2011) Asthma and allergic symptoms and type 1 diabetes-related autoantibodies in 2.5-yr-old children. Pediatr Diabetes 12:604-610

20. Williams AJ, Bingley PJ, Bonifacio E, Palmer JP, Gale EA (1997) A novel micro-assay for insulin autoantibodies. J Autoimmun 10: $473-478$
21. Holmberg H, Vaarala O, Sadauskaite-Kuehne V, Ilonen J, Padaiga Z, Ludvigsson J (2006) Higher prevalence of autoantibodies to insulin and GAD65 in Swedish compared to Lithuanian children with type 1 diabetes. Diabetes Res Clin Pract 72:308-314

22. Vaziri-Sani F, Delli AJ, Elding-Larsson H et al (2011) A novel triple mix radiobinding assay for the three ZnT8 (ZnT8-RWQ) autoantibody variants in children with newly diagnosed diabetes. J Immunol Methods 371:25-37

23. Holmberg H, Wahlberg J, Vaarala O, Ludvigsson J (2007) Short duration of breast-feeding as a risk-factor for beta-cell autoantibodies in 5-year-old children from the general population. Br J Nutr 97:111-116

24. Hermann R, Turpeinen H, Laine AP et al (2003) HLA DR-DQencoded genetic determinants of childhood-onset type 1 diabetes in Finland: an analysis of 622 nuclear families. Tissue Antigens 62: 162-169

25. Ziegler AG, Hummel M, Schenker M, Bonifacio E (1999) Autoantibody appearance and risk for development of childhood diabetes in offspring of parents with type 1 diabetes: the 2-year analysis of the German BABYDIAB Study. Diabetes 48:460-468

26. Trigwell SM, Radford PM, Page SR et al (2001) Islet glutamic acid decarboxylase modified by reactive oxygen species is recognized by antibodies from patients with type 1 diabetes mellitus. Clin Exp Immunol 126:242-249

27. Wiles TA, Delong T, Baker RL et al (2017) An insulin-IAPP hybrid peptide is an endogenous antigen for CD4 T cells in the non-obese diabetic mouse. J Autoimmun 78:11-18

28. Rizzo P, Pitocco D, Zaccardi F et al (2017) Autoantibodies to posttranslationally modified type I and II collagen in Charcot neuroarthropathy in subjects with type 2 diabetes mellitus. Diabetes Metab Res Rev 33:1-6

29. Kaido T, Yebra M, Cirulli V, Montgomery AM (2004) Regulation of human $\beta$-cell adhesion, motility, and insulin secretion by collagen IV and its receptor $\alpha_{1} \beta_{1}$. J Biol Chem 279:53762-53769

30. Triolo TM, Armstrong TK, McFann K et al (2011) Additional autoimmune disease found in $33 \%$ of patients at type 1 diabetes onset. Diabetes Care 34:1211-1213

31. Nakamura M, Yamazaki I, Nakagawa H, Ohtaki S, Ui N (1984) Iodination and oxidation of thyroglobulin catalyzed by thyroid peroxidase. J Biol Chem 259:359-364

32. Orban T, Sosenko JM, Cuthbertson D et al (2009) Pancreatic islet autoantibodies as predictors of type 1 diabetes in the Diabetes Prevention Trial-Type 1. Diabetes Care 32:2269-2274

33. Insel RA, Dunne JL, Atkinson MA et al (2015) Staging presymptomatic type 1 diabetes: a scientific statement of JDRF, the Endocrine Society, and the American Diabetes Association. Diabetes Care 38:1964-1974 